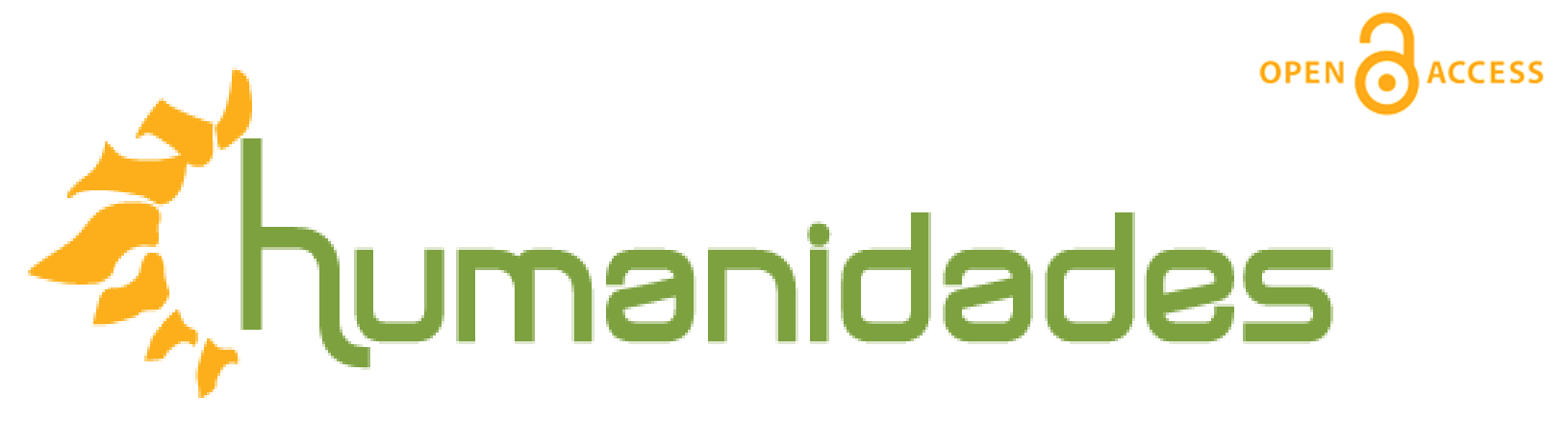

Revista de la Escuela de Estudios Generales, Universidad de Costa Rica

Julio-diciembre, 2016 • Volumen 6, número 2 • EISSN 2215-3934 • pp. 1-31

Recibido: 08-Febrero-2016 Aceptado: 3-Mayo-2016

\title{
Jóvenes, literatura y derechos humanos: Una experiencia en las aulas de Humanidades
}

DOI: http://dx.doi.org/10.15517/h.v6i2.26749

\section{Marisol Gutiérrez Rojas}

Doctora en Educación. Profesora en la Escuela de Estudios Generales, Universidad de Costa Rica.

Correo electrónico: marigu62@hotmail.com 


\title{
Jóvenes, literatura y derechos humanos: Una experiencia en las aulas de Humanidades
}

\section{Resumen}

El artículo registra una experiencia académica con jóvenes universitarios en los cursos de Humanidades de la Universidad de Costa Rica. Cerro Pelón, lágrimas de barro, de María Pérez-Yglesias, es la obra literaria seleccionada para atender tres objetivos puntuales: primero, entender la literatura como una práctica social que participa en la construcción de espacios y entornos del desarrollo humano; segundo, comprender la realidad centroamericana, en especial de las personas jóvenes y desde un enfoque de derechos, a partir de la lectura y análisis de un cuentario cuyos protagonistas son adolescentes y jóvenes; y tercero, ofrecer una experiencia de lectura placentera, dialógica y creativa.

Palabras clave: Literatura latinoamericana, derechos humanos, joven, lectura, Centroamérica.

\section{Young, Literature and Human Rights: An Experience from the Humanities Classes}

\begin{abstract}
This article analize a literary reading experience with Humanity students from the University of Costa Rica. Cerro pelón, lágrimas de barro by María Pérez Yglesias, was the literary piece selected because it clearly covered the three objectives of this academic experience: To see the literature as a social praxis that reflects the space and sourrandings of the human development. To understand the central-american reality, especially the youth, from a humans rights approach, throughout the reading and analysis of these shorth-stories whose characters are mainly teen-agers. To offer the students an experience of refreshing, pleasant, educational, interactive, creative and easy reading.
\end{abstract}


El libro de cuentos Cerro Pelón, lágrimas de barro (2013), de María

Pérez-Yglesias, forma parte de una trilogía compuesta por Las fronteras de la luna y el sol (2008) y Silencio, el mundo tiene el ala rota (2010). De acuerdo con la Dra. Ligia Bolaños Varela, especialista en historiografía literaria centroamericana, este conjunto de obras "constituye el primer ejercicio escritural literario de una costarricense sobre Centroamérica" (L. Bolaños, comunicación personal, 18 de marzo de 2015). Estos tres textos llevan al público lector, como la autora misma señala, "por viejas historias y nuevas realidades", desde la mirada protagónica de niños, niñas y adolescentes.

Durante el segundo semestre de los años 2013 y 2014, en el marco de un curso ofrecido por la Sección de Comunicación y Lenguaje, de la Escuela de Estudios Generales de la Universidad de Costa Rica (UCR), los estudiantes de primer ingreso recibieron un programa de estudios titulado Revelando cosas bajo las palabras y palabras bajo las cosas. Dicho sistema se estructuró en tres unidades: Construyendo con palabras, Más allá de los textos escritos: Trozos y trazos de la región y Revelando palabras.

El curso se propuso crear puentes entre los distintos países de Centroamérica, a través de la literatura, para conocer y entender una temporalidad: décadas de los años setenta y ochenta, en contrapunto con la actualidad nacional y regional de la joven población estudiantil. 
Así, el texto literario central fue Cerro Pelón, lágrimas de barro $^{l} \mathrm{de}$ María Pérez-Yglesias.

La clase se organizó en siete grupos, cada uno de estos asumió el análisis de un cuento y una indagación sobre el país en el cual se desarrolla la historia relatada, de manera que, el contexto social formara parte de la experiencia lectora. ${ }^{2}$ Paralelamente, se le ofrece al estudiantado una gama de películas de o sobre Centroamérica, además, se programaron visitas a galerías con muestras de arte centroamericano, sobre todo de artistas jóvenes, y a la Feria Internacional del Libro, la cual se lleva a cabo durante el segundo semestre del año, por lo que, coincidió con este curso.

La dinámica de trabajo se asentó en el principio de que el conocimiento es una construcción permanente, y de acento lúdico, el cual se nutre con la participación activa de todas y de todos. Por lo anterior, al finalizar cada unidad de trabajo se generó un producto creativo, por ejemplo, viajes por Centroamérica en aviones imaginarios, con pilotos que relataban detalles sobre los paisajes que se podían observar en los videos proyectados en la clase. Así mismo, recorridos por ferias de comida, donde las pupusas, las variedades del gallo pinto, los dulces y los refrescos nacionales les llevaron también a inmiscuirse por primera vez en la cocina. Finalmente, la escritura de nuevos cuentos, como es el caso de Algo más que relatos, sobre el cual se comentará más adelante, 
y la elaboración de un ensayo al final del curso; una producción individual cimentada en un tema específico asignado. ${ }^{3}$

La primera unidad del curso apunta hacia el conocimiento de Centroamérica, ya que el estudiantado actual conoce poco o nada sobre esta región. Si bien a diario se exponen noticias sobre migraciones, pobreza, desastres naturales, Gobiernos corruptos, violación de los derechos humanos, entre otros asuntos que se relacionan con América Central, lo cierto es que gran parte de las personas jóvenes desconoce la zona geográfica en la que viven.

Ciertos temas sobre el istmo son expuestos de manera superficial en la prensa, salvo algunas excepciones; en cualquier caso, resulta importante hacer un esfuerzo por analizar y atender las situaciones que se presentan, especialmente desde espacios educativos formales y desde la misma academia. En ese sentido, deberían fortalecerse los estudios culturales centroamericanos, para entender, por ejemplo, cómo cada persona se construye, se representa y se imagina en la diversidad y en la heterogeneidad que la signa.

Al respecto, el grupo de estudiantes brindó impresiones muy elocuentes. Por ejemplo:

\section{Construyendo con palabras}


Al iniciar el curso, de Centroamérica no sabíamos nada, no más que los países que la conforman; y con algo de vergüenza, debo admitir, que ni siquiera la exacta ubicación geográfica de estos. Poco a poco la fuimos conociendo, y poco a poco también, a través de los relatos, nos hemos llegado a sentir más centroamericanos. Hemos vivido el amor por nuestra tierra y por nuestras naciones hermanas con las cuales compartimos una sangre, una ascendencia; el rostro criollo, mestizo; en mi caso, el rostro moreno, de sangre indígena, de raíces chorotegas. Y el orgullo nos invade el pecho; ese orgullo de saber que somos diferentes del mundo, pero que entre nosotros somos iguales, la misma sangre, el mismo cielo, la misma tierra (P. Angulo, comunicación personal, 2014).

Así las cosas, descubrir que Centroamérica es una región joven, exuberante, diversa y, por sobre todo, en palabras de la clase, "una región encerrada en sus sufrimientos" resulta una experiencia extraordinaria para la gran mayoría. En definitiva, a lo largo del curso, el centro de América creció ante sus ojos, de la misma manera que lo hace la silueta humana que dibujaron el primer día de clases en un pliego de papel. ${ }^{4}$

Para adentrarse en las particularidades del contexto cultural y socioeconómico centroamericano, se parte de una revisión crítica de un 
Informe del Estado de la Región y otros textos atinentes. Lo anterior se realiza desde un enfoque de derechos que, en consonancia con la Política Pública de la Persona Joven (2004), reconoce a las personas como sujetos de derechos en el marco de una "concepción de integralidad, no discriminación, equidad e igualdad social, y favorece a la totalidad de las personas" (p. 25).

Este recorrido pone al estudiantado frente a un pequeño territorio que forma parte de la superficie terrestre mundial $\left(523780 \mathrm{~km}^{2}\right)$, el cual posee extraordinarias reservas naturales y una diversidad desbordante; no obstante, alberga también conglomerados humanos que reclaman una vida "vivible". Al respecto, el Informe del Estado de la Región muestra porcentajes significativos de personas para quienes el acceso a servicios básicos como el agua potable y la electricidad siguen siendo una quimera.

Ahora bien, la revisión de documentos generados por la Organización Iberoamericana de Juventud y de algunos informes sobre el estado de los derechos de la niñez y la adolescencia en la región, pone a los estudiantes en contacto con las políticas sociales destinadas a estas poblaciones. Asimismo, les permite constatar que, a pesar de los esfuerzos regionales realizados (conferencias, declaraciones, acuerdos, convenios y códigos), la población menor de edad se encuentra en un estado de significativa vulnerabilidad. 
Las reflexiones sobre este material se hilvanan con una serie de películas sobre y de Centroamérica: Voces inocentes (2004), El camino (2008), El regreso (2012), Sin nombre (2009), Princesas rojas (2013) y Romero (1989). Estas, además, se enlazan con la visita a la Feria Internacional del Libro, ${ }^{5}$ a galerías y otros centros de arte como TEOR/éTica. ${ }^{6}$ En el fondo, estas actividades les permiten enfrentarse a otros datos, otras voces, otras sensibilidades de artistas jóvenes que les describen el paisaje agridulce de la Centroamérica heredada que hoy habitan.

Este recorrido les lleva a plantearse cuál es el papel de las políticas culturales de la región en tanto promotoras y defensoras de los derechos humanos, de igual manera, en el logro de una visión compartida de Centroamérica. Pues bien, una y otra vez, pueden constatar que la precaria inversión regional en cultura y recreación se traduce en débiles o ausentes políticas para este sector y en ministerios de cultura pobres, inexistentes o unidos a otras entidades, cuyo accionar apunta hacia otros intereses -como es el caso de la educación y el turismo-, lo cual genera que los exiguos presupuestos sean distribuidos (o diluidos) en tareas disímiles.

La centralización de las acciones en las ciudades es otro asunto que llama su atención, puesto que se reproducen políticas hegemónicas y 
excluyentes que desfavorecen las zonas costeras y rurales, los grupos étnicos y las diversidades culturales.

En el marco de las discusiones sobre la cultura como un derecho humano, los estudiantes llegan a entender el papel de la literatura como un medio útil y valioso para conocer la realidad de los pueblos y de grupos poblacionales específicos.

La segunda unidad del programa lleva a los conversatorios sobre Cerro Pelón, lágrimas de barro. Este texto contiene seis cuentos que aluden a los países centroamericanos, a excepción de Belice. Para cubrir esta nación, se recurre al libro Las fronteras de la luna y el sol, específicamente, a la lectura "Isidra, el canto de tu sirena".

Cerro Pelón, lágrimas de barro aparece ante sus ojos como una metáfora de la erosión que se produce en la condición humana, del desgaste que sufren las calidades que signan a cada persona como ser humano. Se trata de una metáfora de Centroamérica, de su gente, de la continua violación de los derechos humanos y de su desesperanza, la cual surge ante los juegos de poder diseminados en la institucionalidad y ante aquellos sucesos que se transforman en desastres naturales por la falta de políticas ambientales. Sin embargo, el texto también se presenta como una oportunidad para re-conocer-

\section{Más allá de los textos escritos: \\ Trozos y trazos de la región}


se y "para evidenciar la necesidad de una nueva visión de vida", tal cual lo plantea un estudiante.

El primer señalamiento que hace el grupo sobre la lectura se relaciona con la condición etaria de quienes protagonizan las historias que conforman el cuentario Cerro Pelón, lágrimas de barro. Todos los personajes principales son adolescentes y jóvenes que, como se declara en la presentación del libro, "asumen su derecho a la palabra y la expresión".

$\mathrm{Al}$ revisar previamente los indicadores sociales de Centroamérica, el estudiantado ya sabe que esta población representa un porcentaje significativo y que es altamente vulnerable. Además, por experiencia personal, reconocen que los espacios para la palabra y la expresión son escasos durante esta etapa de la vida. De hecho, como se evidencia y se discute en el curso, la literatura escrita sobre, para y por esta población es casi nula.

Por lo expuesto hasta ahora, el cuentario provocó suma empatía entre quienes lo leyeron. Valga la siguiente cita para ilustrar este punto:

Hay miles de adolescentes con sueños e ilusiones, al igual que los protagonistas de cada cuento del texto literario base del curso. Luchadores sagaces, pero que por distintas razones de la vida son 
marginados, silenciados, empobrecidos, pero no dejan de lado la esperanza de que en algún momento las oportunidades llegarán. Y si un texto literario puede provocar enojo, tristeza e indignación por lo que expone, cuántas realidades de esos jóvenes podrían cambiar si como actores sociales que somos buscamos la mejor manera de involucrarnos en el cambio desde las mismas comunidades (J. Monge, comunicación personal, 2014).

Para algunos estudiantes, este libro es "un diario" que muchos centroamericanos, víctimas de abuso, no pudieron escribir; y una oportunidad invaluable para entender el significado de "nosotros". En palabras de jóvenes universitarios, se señala el hecho de que la sociedad presta más atención al "nos" que al "otros"; los "otros" se ignoran, se desconocen y, en consecuencia, se temen.

Cada cuento es una exploración humana particular. "Jachel, la perra muda", texto con el que se inaugura el libro, fue uno de los cuentos más comentados en la clase. Este se sitúa en Guatemala, relata la historia de las hermanas Gertrudis y Marcia, unas niñas de 13 y 10 años, respectivamente. Ellas presencian la destrucción de sus pueblos indígenas, en una ocasión, provocada por los paramilitares y, en otro momento, a manos de los militares. 
Huimos del silencio. Yo me llevo por dentro un montón de chispas para encender la cólera, la rabia, el rencor cada vez que lo necesite. El fuego me hace más fuerte. La gente supone que estamos de duelo, sabe que no podemos llevar al cementerio, para que descansen en paz, a las personas que más nos quisieron. Sabe que, aunque duele el alma, las palabras se esfuman para no delatar.

Marcia me Mira con ojos de asombro. Me toma la mano con ternura y acaricia mi mutismo. Jachel camina con el rabo entre las patas. Triste, parece que al fin descubrió que no puede ladrar. Muda, olfatea el camino para avisarnos del peligro. Siente el retumbar de la callecilla de tierra para descubrir si la golpean las botas militares. Observa cualquier movimiento, mira a lo lejos y nos lame las manos con ternura.

Marcia, la única de las tres que alegraba al aire, calla. Callamos y esperamos un nuevo día (Pérez-Yglesias, 2013, pp. 20-21).

El derecho a la palabra -tan apreciado por las personas jóvenes- se reclama a través de metáforas del silencio (representado por Jachel) y de la presencia/ausencia de la "gente del silencio". Lo anterior conmueve e indigna a la comunidad de jóvenes lectores. 
"Cerro Pelón, lágrimas de barro", cuento que da título al libro, se desarrolla en una zona rural costarricense, donde la deforestación y la explotación de un tajo, en las entrañas de un cerro, se tiñen de intereses políticos y comerciales. Esta situación provoca una gigantesca tragedia ambiental y humana, la cual ni el adolescente amor de Agustín y Beatriz puede detener.

Cerro Pelón agoniza en medio de la tormenta. Lo presiente. Lo sabe. Observa cómo se anegan las raíces amputadas, los arbustos que se desgajan al arrastrar los troncos maderables, el suelo arrasado por el machete indiferente de los extraños. Siente su vientre frágil, vacío de piedra.

Cerro Pelón pierde también el último verdor de la esperanza (Pérez-Yglesias, 2013, p. 47).

La tensión entre la esperanza y la desesperanza interpela a los estudiantes, les atañe y les lleva a reconocer su compromiso en la toma de decisiones sobre el tema ético-ambiental.

"Y ahora el Mitch", tercer cuento del libro, relata la exclusión social, la explotación sexual comercial de personas menores de edad, la desintegración familiar y los desastres naturales (el huracán Mitch), en un país (Honduras) atormentado por la violencia sistemática de todo 
orden. El protagonista Miguel (Migue, Lito, Lillo o Micky) sufre tanto dolor que solo quiere "volar lejos, encima de las ruinas grises de Copán" (Pérez-Yglesias, 2013, p. 83).

“¿Quién es Miguel?”, se pregunta un estudiante, luego, él mismo se responde:

Para mí no es más que la imagen que tenemos los centroamericanos de nosotros mismos desde los ojos del otro; de lo que debemos pensar de nosotros mismos desde lo que otros nos dicen que somos... El nombre refleja aquello que entendemos y la relación que tenemos con la cosa nombrada. Nombres foráneos (América y Micky) para poblaciones, regiones, personas que ya tienen uno, pero a quienes se les ha atropellado su identidad (N. Espinoza, comunicación personal, 2014).

El cuento "Una mañana azul" lleva a sus jóvenes lectores a una Nicaragua imbuida en una protesta social (en defensa de la tierra) que se ve alterada por un violento sismo y un huracán. De nuevo, la esperanza, la solidaridad y el amor toman cuerpo en una figura adolescente; esta vez, se trata de una colegiala. Ella, al igual que Mafalda (la famosa tira cómica argentina), expresa: "Paren el mundo, quiero bajarme". 
Con ojos de nostalgia nos cuenta que se vino de su pueblo al nuestro atrapado por el amor futuro de una morena risueña y escandalosa que le devuelve las ganas de vivir. Nos reímos con el chiste. Nos habla sobre la represión dentro de la guerrilla de cada país, la pobreza en que se vive, lo que significa no tener democracia, la maravilla de las culturas indígenas, la forma cómo venden las tierras a los extranjeros, el miedo a la cárcel, a la desaparición, al exilio obligado... Tengo miedo.

"Paren el mundo que quiero bajarme".

Miro el globo de la tierra igual que Mafalda y pienso en los chinos roncando. Trato de imaginarme lo que sería vivir con un temor permanente, sin final (Pérez-Yglesias, 2013, p. 101).

El penúltimo cuento, "Muerte en la calle", alude a la resistencia organizada en El Salvador aguerrido, desde la mirada comprometida de una pareja de jóvenes estudiantes universitarios, radicados en suelo europeo. La muerte violenta, su amor, sus sueños y la actitud de la familia ponen en evidencia no solo la corrupción política, sino también la doble moral de las clases acomodadas.

El grupo de estudiantes rescata, de este mural siniestro, la valentía de sus pares, no ajena, por lo demás, a la aguda autocrítica, como 
puede verse en las líneas escritas por una estudiante en su ensayo de final de curso:

Las personas jóvenes somos una vez más clave en los movimientos de protesta. Costa Rica ha sido testigo de cómo los estudiantes universitarios salimos a las calles a defender, cuestionar o declararnos en contra. Sin embargo, como dice la protagonista de la historia: "No sé si la ignorancia permite dormir mejor, comer bien, pensar tranquilamente" (Pérez-Yglesias, 2013, p. 125).

$\mathrm{Al}$ inicio del semestre, cuando se nos preguntó qué significaba para nosotros Centroamérica y qué sabíamos sobre la gente de nuestra edad, el silencio en unos y la vacilación en otros, dejó como conclusión que para la mayoría esas preguntas no habían formado parte de las reflexiones. Y es que ser centroamericano no es solamente vivir en un mismo pedazo de tierra, es formar parte de una historia... Miriam en uno de los párrafos del texto dice: "Pensamos en los amigos y las amigas costarricenses que parecen venir de un mundo diferente -casi de otro planeta-... José Eduardo y Ana confiesan, ingenuamente ticos, que es desde estas tierras, al otro lado del mar, que empiezan a percibir el continente latinoamericano, a conocer el istmo centroamericano con sus habitantes y tragedias, que aprenden a matizar su patria de asilo sin ejército (Pérez-Yglesias, 2013, p. 125). Al vivir en un 
país que se considera pacífico, sin ejército, es normal que no se valore lo que se tiene, pero ¿es aceptable llegar a este punto de egocentrismo e indiferencia por lo que pasa en el país vecino? (M. Naranjo, comunicación personal, 2014).

Por último, "Jesibú" traslada, al joven grupo de lectores, a una Costa Rica limítrofe, multicultural, sincrética e inundada por las aguas del río Sixaola. El maltrato hacia las mujeres (niñas y adultas), en particular el incesto, se enfrenta a la resistencia de la esperanza y a la confianza de lograr un mundo distinto:

Estoy convencida que un día de estos, cuando los recuerdos se limpien como las huellas de la inundación, dejaré de temerles a los hombres y, como una luna llena capaz de desbordar el agua, pariré mi primer hijo. Tendré un niño con sonrisa de dientes blancos y ojos negros, como los míos. Un pequeño de los que hay que proteger más porque son más débiles cuando chiquitos. Pariré un güila que me va a cuidar y a querer cuando se convierta en hombre. Un hijo del cielo sin nubes y de la tierra fértil. Jesibú (Pérez-Yglesias, 2013, p. 172).

A pesar de los dolores narrados, los estudiantes concluyen que Centroamérica y su población joven no están "sentenciados al 
sufrimiento" y que son, precisamente las personas jóvenes, quienes construyen y mantienen la esperanza con amor y solidaridad.

En buenas cuentas, la interpelación de la realidad "externa" que permite este texto literario es también para la clase una interpelación a sí mismos, en tanto se identifican con las personas jóvenes que viven los sucesos y ponderan su papel como agentes de cambio; no así como reproductores de sistemas excluyentes y violatorios de los derechos humanos. Las siguientes palabras subrayan lo mencionado antes:

Este libro me impactó profundamente. Yo sabía de los sucesos ocurridos en la región, como el desastre provocado por el Mitch en Honduras o la guerra civil salvadoreña, incluso tengo amistades que se vieron afectadas por esta, pero nunca había logrado identificarme con la juventud de la época que tuvo que sufrir y superar tales hechos. En este caso la literatura es el medio que propicia la unidad entre los pueblos centroamericanos. Lo que les pase, será lo que nos pase a nosotros, porque esta es nuestra tierra y nuestra gente, y ya nadie se quedará de brazos cruzados (A. Chacón, comunicación personal, 2013).

El libro, entonces, cumple con su finalidad comunicativa, estética, social (de conocimiento, denuncia y toma de conciencia) y, sobre todo, actúa como motor de cambio. De esa manera lo evidencia una 
estudiante, quien al finalizar el curso declara: "ser estudiante universitaria es pertenecer al universo; no cegarnos ante lo que ocurre alrededor, al contrario, luchar para que lo que veamos sea mejor para todos" (J. Monge, comunicación personal, 2014). Para aquellos que laboran en el ámbito educativo, esto es, sin duda, un importante indicador de aprendizaje.

La última unidad del programa del curso conduce al encuentro con

\section{Revelando palabras} la autora del texto leído. María Pérez-Yglesias es una mujer de la academia, a esta ha dedicado su vida laboral y afectiva. Actualmente, ya jubilada, recuerda su tránsito por las aulas universitarias como docente de literatura y como comunicadora y semióloga, tarea que nutre con más de un centenar de publicaciones académicas. Posteriormente, destina sus afanes hacia importantes puestos de dirección en la Universidad de Costa Rica, por ejemplo, como Decana del Sistema de Estudios de Posgrado y como Vicerrectora de Acción Social. Ya madura, amplía los intereses de su experiencia vital y se introduce en la escritura de ficción, donde la versatilidad y capacidad comunicativa, que cautiva a sus estudiantes, se transforma en una copiosa producción de cuentos, relatos, novelas y memorias.

Los conversatorios entre ella y los grupos de estudiantes resultan emotivos, elocuentes y críticos. No falta el señalamiento al papel de la memoria; cómo esta cuenta, pesa y vale. En palabras de la clase: 
"la historia está hecha para recordar y aprender de ella, para tener un mejor presente y un futuro posible". Por eso, hablan sobre los derechos humanos, cómo estos se han convertido en un discurso que suele saltar a la palestra cuando las violaciones toman el escenario.

Así mismo, durante estos conversatorios, los estudiantes subrayan la actuación de adolescentes y jóvenes como solidarios personajes de las historias. Lo anterior les hace pensar que, a pesar de las tragedias que viven, no están condenados; tienen posibilidades de salir adelante como protagonistas de su propia experiencia, como agentes de cambio. Se interesan, además, en saber por qué la escritora elije a esta población para hablar de Centroamérica y por qué se interesa precisamente en este espacio geográfico.

$\mathrm{Al}$ respecto, la autora señala que el ejercicio de la escritura está marcado por historias de vida, por las experiencias y los quehaceres particulares y sociales. En ese sentido, agrega que sus responsabilidades académicas le han permitido viajar y permanecer por días en cada uno de los países de esta región, la cual quiere y por la cual se conduele. Educadora al fin, Pérez-Yglesias sostiene que:

Para escribir hay que observar y escuchar, porque la literatura está hecha de detalles y no de grandes trascendencias. Para escribir es necesaria esa pasión que hay que guardar toda la vida, pero que 
palpita de manera especial en la adolescencia, una edad en la que, además, aún permanece la curiosidad de la niñez" (M. PérezYglesias, comunicación personal, 11 de noviembre de 2014).

Momentos altamente emotivos se vivieron en estos conversatorios. En el año 2013, la primera vez que se imparte el curso, un grupo de estudiantes le entrega a la autora una respuesta escrita sobre la lectura de Cerro Pelón: lágrimas de barro, la cual titularon Algo más que relatos. Se trata de seis narraciones breves escritas por estudiantes oriundos de Curridabat, Heredia, Puriscal, Pavas y San Carlos. Estas constituyen un ejercicio de historia local con protagonistas tomados de sus comunidades, de igual manera, representan una suerte de puentes entre los trozos y trazos de una Costa Rica bastante desconocida por algunas personas.

En el año 2014, un estudiante hondureño, radicado en Costa Rica, expresó su emoción al leer un libro que le permite pensar en su identidad, reencontrarse con sus padres y dialogar con ellos y con la familia que aún vive en su país de origen. En otra clase, una estudiante del año anterior expuso al nuevo grupo y a la autora su ensayo titulado Jachel y la perra muda: más que una metáfora. Un hermoso análisis de las figuras literarias presentes en este cuento, desde su condición de estudiante de Farmacia; lo cual se convierte un interesante ejercicio de interdisciplinariedad. 
Por último, un estudiante muy interesado por el cine le comentó a la autora su enorme emoción al haber leído, por primera vez en su vida, un texto literario; experiencia que nunca antes había cumplido, ni por obligación ni por placer. Agrega que esta experiencia fue como vivir "seis películas distintas", lo cual le complace y le hace pensar en futuras lecturas. Esta declaración la registra luego en su ensayo de final de curso, con las siguientes palabras:

Cuando le abres la puerta de tu hogar a una persona, dejas entrar su historia, sus anécdotas, sus hábitos, sus miedos. Quizás por eso le echaba el pestillo a la puerta; razón por la cual crecí sin conocer ni siquiera el cantón donde habito.

A mis 20 años, este es el primer libro que he leído. Aunque la palabra "leído" se queda corta a la hora de describir la experiencia adquirida con su lectura, los lugares a los que fui transportado, las emociones transmitidas, las diversas situaciones y problemáticas que viven los adolescentes y jóvenes de mi región, conocer lo desconocido y vivir lo nunca vivido... En un momento así, cuando le hemos dado la oportunidad a aquello que nos disgusta, y nos damos cuenta de lo maravilloso que resultó ser, por nuestra cabeza naturalmente pasan dos pensamientos: el lamentarse por el tiempo perdido o el alegrarse de haberlo dejado entrar a tu vida y querer 
recuperarlo. Por mi parte, me quedo con el segundo pensamiento (K. Hidalgo, comunicación personal, 2014).

Los encuentros al final del curso suponen un ejercicio de evaluación Síntesis para unir y poner en diálogo todas las partes discutidas, analizas y vividas alrededor del texto literario $\mathrm{y}$ de las realidades centroamericanas: las de ayer y las de hoy. El viaje por Centroamérica, realizado por la clase, abre un espacio para reflexionar sobre los productos generados y para responder, además, algunas interrogantes asociadas con la vivencia que cada uno y cada una ha tenido con la literatura y con los procesos de lectura.

De esta forma, se concluye que el encuentro con la literatura debe ser un diálogo placentero y no un enfrentamiento o una imposición. Se reconoce que la escogencia de Cerro Pelón, lágrimas de barro, entre una gran oferta literaria para esta experiencia educativa, fue certera. El texto posee cualidades altamente atractivas para los lectores jóvenes: brevedad; una trama ágil, vertida en una prosa de ribetes poéticos, escritos en primera persona; y la presencia de ilustraciones ${ }^{7}$ que subrayan, dialogan e interpelan las historias.

Así mismo, aunque las historias narran situaciones difíciles para los protagonistas y violentos sucesos naturales y sociales, estos no dejan de ser afines a esta comunidad de lectores. Lo anterior porque los 
personajes son cercanos a su edad, a sus intereses, a sus anhelos y preocupaciones; en consecuencia, la apropiación del texto es posible y de esto dan cuenta los ensayos escritos a partir de la lectura. El siguiente fragmento permite ilustrar este punto:

Esa obra que parte a parte fue escrita, parte a parte dejó en nuestros corazones el horror plasmado de las realidades que se viven en nuestros países hermanos. Al leerla, solo, en mi habitación, me transportaba a una pequeña comunidad hondureña, al sótano de una iglesia nicaragüense, al Cerro Verde, a ese cerro que imaginaba hermoso, boscoso, un pedacito de pulmón terrestre, y que hombres inescrupulosos acabaron con él... Yo he quedado con la espinita del saber, con esas ganas de aprender más, de viajar, de conocer, de buscar entre la maleza, entre las cicatrices de "un pueblo sin piernas, pero que camina" (Calle 13, 2010), y ojalá, ojalá encuentre la inspiración que he sentido leyendo sobre la tierra que nos ha visto crecer, la tierra que nos da de comer, que nos da abrigo, y nos da el lugar preponderante que tenemos entre los lugares más bellos del mundo (P. Angulo, comunicación personal, 2014).

Aunado a lo anterior, la mediación pedagógica y didáctica favoreció el desarrollo afectivo y cognitivo, lo cual aumentó el interés por los demás y la solidaridad, así como el análisis crítico y propositivo. Esto coincide 
con lo que María Pérez-Yglesias, en sus conversatorios, denominó “el paso del paradigma de la competencia al paradigma del complemento y la colaboración".

Este paradigma es evidente en la forma en que se abordaron los temas de los cuentos y en lo vivido durante las clases. La participación activa de las voces, las ideas y las miradas ayudaron a imaginar una forma de vida en la cual se respetan los derechos humanos y las diferencias. En su ensayo final, un joven estudiante señaló al respecto:

El dolor no quedó en el pasado, vive en nuestro presente, en nuestra gente, tal y como si el dinosaurio todavía estuviera aquí, entre nosotros. ${ }^{8}$ Lo difícil es pasar del papel y las palabras al acto, y esa es nuestra responsabilidad como ciudadanos de la zona: despertar y eliminar al dinosaurio. Qué difícil suena, difícil para un grupo de jóvenes soñadores, dirían; pero siento que es mi responsabilidad conocer y dar a conocer cómo vive nuestra gente... Centroamérica es más que pequeños países que han sido violentados y lo siguen siendo; es una de las zonas más bellas del planeta y habitada por seres humanos que merecen ser tratados como tal (J. Elizondo, comunicación personal, 2014).

Si bien aquellas siluetas de los cuerpos (dibujadas al inicio del curso) continúan fragmentadas, ahora exhiben indicadores sociales 
centroamericanos, rostros y paisajes capturados en fotografías; poemas escritos por el grupo y por poetas de la región. Así mismo, algunos colores de sus banderas; trozos de canciones ticas, nicas, catrachas, guanacas, chapinas y canaleras; parte de sus artesanías, tejidos y bordados; e imágenes de sus danzas y de sus instrumentos.

Las siluetas mudas e inertes trastocan el luto, el dolor y la destrucción, para dar paso a una única frontera solidaria y esperanzadora: la frontera de la luna y el sol, aquella hermosa expresión poética y reveladora que da nombre a otro de los cuentarios de María Pérez-Yglesias. 
Jóvenes, literatura y derechos humanos...

A Band Apart, Lawrence Bender Productions, Altavista Films, MUVI Films y Referencias Organización Santo Domingo. (Productor) y Mandoki, L. (Director). (2004). Voces Inocentes [Película]. Estados Unidos, México y Puerto Rico.

Agosín, M. (1989). La literatura y los derechos humanos. Aproximaciones, lecturas y encuentros. San José, Costa Rica: EDUCA.

Astarté Producciones y Gedeon Programmes. (Productor) y Yasin, I. (Director). (2008). El camino [Película]. Costa Rica.

Canana Films, Creando Films y Primary Productions. (Productor) y Fukunaga, C. (Director). (2009). Sin nombre [Película]. Estados Unidos y México.

Champeil, V. (2010). El derecho a la cultura como derecho fundamental. Revista Electrónica Iberoamericana, 4(1), 92-116.

Chavarría, G. (2010). Literatura y humanismo en el siglo XXI. San José, Costa Rica: ALICAC.

Consejo Nacional de la Niñez y Adolescencia, Universidad de Costa Rica y Fondo de las Naciones Unidas para la Infancia y (2011). VII Estado de los Derechos de la niñez y adolescencia en Costa Rica. Hacia el cumplimiento de la política nacional para la niñez y adolescencia (2009-2021). Recuperado de http://www.unicef.org/costarica/docs/cr_pub_EDNA_VII_CR.pdf

Consejo Nacional de la Política Pública de la Persona Joven. (2004). Política Pública de la Persona Joven. San José, Costa Rica: Ministerio de Cultura y Juventud. 
Galich, F. (1998). Reflexiones en torno a los problemas para una teorización e historización de la literatura centroamericana. Managua: IHNCA-UCA.

Hol y Asociados S.A., Suécinema C.A. y La Feria Producciones. (Productor) y Astorga, L. (Director). (2013). Princesas rojas [Película]. Costa Rica.

Jiménez, A. (2004). Filosofía, literatura y memoria de las víctimas. Revista Herencia, 16 (1-2), 53-63.

Klisksberg, B. (2014). ¿Cómo enfrentar la pobreza y la desigualdad? Una perspectiva internacional. San José, Costa Rica: Editorial Universidad de Costa Rica.

Miel y Palo Films. (Productor) y Jiménez, H. (Director). (2012). El regreso [Película]. Costa Rica.

Monterroso, A. (1959). Obras completas (y otros cuentos). España: Anagrama.

Organización Iberoamericana de Juventud. (2011). Políticas de juventud en Centroamérica. Construyendo un paradigma para el desarrollo social. Recuperado de http://www.oij.org/es_ES/publicacion/politicas-de-juventuden-ca-construyendo-un-paradigma-para-el-desarrollo-social

Paulist Pictures (Productor) y Duigan, J. (Director). (1989). Romero [Película]. Estados Unidos.

Pérez-Yglesias, M. (2008). Las fronteras de la luna y el sol. San José, Costa Rica: EUNED. 
Jóvenes, literatura y derechos humanos...

Pérez-Yglesias, M. (2013). Cerro pelón, lágrimas de barro. De adolescentes y desastres en Centroamérica. España: Centro Extremeño de Estudios y Cooperación con Iberoamérica.

Pérez-Yglesias, M. (2013). Cerro pelón, lágrimas de barro. San José, Costa Rica: EUNED.

Programa Estado de la Nación. (2011). Cuarto Informe Estado de la Región en Desarrollo Humano Sostenible. San José, Costa Rica.

Sandoval, C. (2002). Otros amenazantes. Los nicaragüenses y la formación de identidades nacionales en Costa Rica. San José, Costa Rica: Editorial de la Universidad de Costa Rica. 
1. Durante el curso se trabajó con la edición de la EUNED (2013). La primera edición de este libro se realizó en España, durante el año 2010, bajo el sello editorial del Centro Extremeño de Estudios y Cooperación con Iberoamérica (CEXECI) y con el título Cerro Pelón, lágrimas de barro. De adolescentes y desastres en Centroamérica.

2. Cerro Pelón, lágrimas de barro no incluye a Belice, por lo tanto, se acudió a otro cuentario, Las fronteras de la luna y el sol, donde la historia "Isidra, el canto de tu sirena" se desarrolla en tierra beliceña.

3. Algunos fragmentos de estos trabajos ilustran este artículo.

4. Como actividad inicial, se les solicita delinear el contorno de la figura de un compañero o compañera. Por las dimensiones del papel, solo es posible trazar una parte del cuerpo de la persona que reposa sobre el pliego. El cuerpo, entonces, aparece fracturado, tal como la Centroamérica que posteriormente conocerán.

5. Feria Internacional del Libro en su decimocuarta (2013) y decimoquinta edición (2014). Este espacio les permitió dialogar personalmente con escritores como Gioconda Belli, Sergio Ramírez, Julio Escoto y la misma María Pérez-Yglesias. También, pudieron tocar los libros, escuchar algunas lecturas y conocer la producción centroamericana. Sorprendentemente, la mayoría asistía por primera vez a esta actividad, incluso, algunos ignoraban completamente la existencia de este evento.

6. La visita a la exposición La generación del encierro, una muestra de arte salvadoreño que albergó TEOR/éTica en el año 2014, inquietó mucho a la clase, pues pudieron percibir las secuelas de lo ocurrido en la historia reciente de Centroamérica. Sin ser planificado, ese día se realizó, además, un recorrido por el Barrio Amón, lo cual se convirtió en una valiosa oportunidad para conocer, en compañía de sus pares, un rincón de la ciudad capital que relata en sus edificaciones, muros y plazas, sucesos culturales e históricos importantes de Costa Rica. 
Jóvenes, literatura y derechos humanos...

7. La edición de la EUNED (2013) está ilustrada por Eddy Castro Rojas. Artista autodidacta que ha acompañado varias de las publicaciones de María PérezYglesias.

8. En alusión al cuento del escritor hondureño-guatemalteco Augusto Monterroso, leído en clase.

\section{¿Cómo citar este artículo?}

Gutiérrez, M. (Julio-diciembre, 2016). Jóvenes, literatura y derechos humanos: Una experiencia en las aulas de Humanidades. Revista humanidades, 6(2), 1-31. doi: dx.doi.org/10.15517/h.v6i2.26749 